\title{
Burden of severe illness associated with laboratory confirmed influenza in adults aged 50-64 years: a rapid review
}

\author{
Dong Kim ${ }^{1}$, Allison McGeer ${ }^{1}$, Elizabeth Uleryk $^{2}$, and Brenda Coleman ${ }^{1}$ \\ ${ }^{1}$ Sinai Health System \\ ${ }^{2}$ Elizabeth M Uleryk Consultng
}

December 5, 2021

\begin{abstract}
Background: While the high burden of illness caused by seasonal influenza in children and the elderly is well-recognized, less is known about the burden in adults 50-64 years of age. The lack of data for this age group is a key challenge in evaluating the cost-effectiveness of vaccination programs. We aimed to assess influenza-associated hospitalization and mortality rates, and case fatality rates for hospitalized cases among adults aged 50-64 years. Methods: This review was conducted according to the PRISMA: we searched MEDLINE, EMBASE, Cochrane, Web of Science, and grey literature for articles and reports published since 2010. Studies reporting rates of hospitalization and/or mortality associated with laboratory-confirmed influenza among adults 50-64 or 45-64 years of age for the 2010-11 through 2019-20 seasons were included. Results: Twenty studies from 13 countries were included. Reported hospitalization rates associated with laboratory-confirmed influenza 5.7 to 112.8 per 100,000 . Rates tended to be higher in 2015-2019 compared to 2010-2014, and were higher in studies reporting data from high income versus low and middle-income countries. Mortality rates were reported in only one study, with rates ranging from 0.8-3.5 per 100,000 in four different seasons. The case fatality rate among those hospitalized with influenza, as reported by population-based studies, ranged from $1.3 \%$ to $5.6 \%$. Conclusions: Seasonal influenza imposes a significant burden of morbidity in adults $50-64$ years of age, but with high heterogeneity across seasons and geographic regions. Ongoing surveillance is required to improve estimates of burden to better inform influenza vaccination and other public health policy.
\end{abstract}

\section{Hosted file}

Rapid review Influenza burden in 50-64 year olds nov 162021 submission final.docx available at https://authorea.com/users/449624/articles/548114-burden-of-severe-illnessassociated-with-laboratory-confirmed-influenza-in-adults-aged-50-64-years-a-rapid-review 